\title{
Mind the Gap - Passenger Arrival Patterns in Multi-agent Simulations
}

\author{
Andreas Neumann $^{1 *}$, Ihab Kaddoura ${ }^{2}$ and Kai Nagel ${ }^{2}$ \\ ${ }^{1 *}$ Senozon Deutschland GmbH, Schillerstrasse 112, 12305 Berlin, Germany \\ ${ }^{2}$ Technische Universität Berlin, Transport Systems Planning and Transport \\ Telematics, Salzufer 17-19, 10587 Berlin, Germany \\ 'neumann@senozon.de
}

\begin{abstract}
In most studies mathematical models are developed finding the expected waiting time to be a function of the headway. These models have in common that the proportion of passengers that arrive randomly at a public transport stop is less as headway in-creases. Since there are several factors of influence, such as social demographic or regional aspects, the reliability of public transport service and the level of passenger information, the threshold headway for the transition from random to coordinated passenger arrivals vary from study to study. This study's objective is to investigate if an agent-based model exhibits realistic passenger arrival behavior at transit stops. This objective is approached by exploring the sensitivity of the agents' arrival behavior towards (1) the degree of learning, (2) the reliability of the experienced transit service, and (3) the service headway. The simulation experiments for a simple transit corridor indicate that the applied model is capable of representing the complex passenger arrival behavior observed in reality. (1) For higher degrees of learning, the agents tend to over-optimize, i.e. they try to obtain the latest possible departure time exact to the second. An approach is presented which increases the diversity in the agents' travel alternatives and results in a more realistic behavior. (2) For a less reliable service the agents' time adaptation changes in that a buffer time is added between their arrival at the stop and the actual departure of the vehicle. (3) For the modification of the headway the simulation outcome is consistent with the literature on arrival patterns. Smaller headways yield a more equally distributed arrival pattern whereas larger headways result in more coordinated arrival patterns.
\end{abstract}

Keywords: Multi agent simulation, Passenger arrival pattern, Time adaptation, Service reliability, Headway, Multinomial logit, Evolutionary algorithm, KISS principle, Public Transport, MATSim

\section{Introduction}

The relation of public vehicle and passenger arrivals at stops is approached by several researchers [1-4]. In most studies mathematical models are developed finding the expected waiting time to be a function of the headway. These models have in common that the proportion of passengers that arrive randomly at a public transport stop is less as headway increases. Since there are several factors of influence, such as social demographic or regional aspects, the reliability of public transport service and the level of passenger information, the threshold headway for the transition from random to coordinated passenger arrivals vary from study to study and ranges from 5 to 12 minutes [4].

In macroscopic simulation packages like VISUM, trips are generated from origindestination matrices valid for a time slice, e.g. from 8 a.m. to 9 a.m. Among all 
paths starting within that time slice, the trip router then searches for the least cost path. The trip assigned to that path will start immediately with the path's departure time. The router assumes that the passenger represented by the trip will adapt to the path regardless of the actual position of the path's departure time within the time slice [5]. Due to the lack of activities within four-step models, this is a valid approach, but the trip assignment heavily depends on the combination of the size of time slices and the provided service frequency of the public transport system. More important, these models lack the possibility to simulate realistic arrival patterns at the transit stop.

In this study, we examine how different passenger arrival patterns can be incorporated into the multi-agent transport simulation MATSim. We use a simple time adaptation approach that allows agents to adjust their activity scheduling decisions, e.g., to shorten, extend and shift activities. Our main objective is to investigate if the model exhibits realistic passenger arrival patterns at transit stops. This overall objective is approached by exploring the sensitivity of the agents' behavior. Different simulation experiments are carried out for a simple corridor scenario to investigate three of the above mentioned factors of influence. (1) The agents' degree of learning: We analyze under which conditions the agents overadapt resulting in unrealistic user behavior. (2) The reliability of the experienced transit service: We investigate how the reliability of the experienced departure times affects passengers' travel behavior. (3) The impact of public transport headways. We examine how passengers' arrival patterns change with the headway, i.e., if the transition from random to coordinated arrival patterns can be observed in the model.

\section{Methodology}

This section describes the general simulation approach of MATSim (Section 2.1) and the special characteristics of public transport in MATSim (Section 2.2). Since the method- ology remains unaltered these two sections are based on [6]. Furthermore, Section 2.3 explains the agents' departure time adaptation in MATSim that is of particular impor- tance in this paper. For further information of the simulation framework MATSim, see [7].

\subsection{MATSim Overview}

In MATSim, each traveler of the real system is modeled as an individual agent. The approach consists of an iterative loop that has the following steps:

1. Plans Generation: All agents independently generate daily plans that encode among other things their desired activities during a typical day as well as the transport mode for every intervening trip.

2. Traffic Flow Simulation: All selected plans are simultaneously executed in the simulation of the physical system. The traffic flow simulation is implemented as a queue simulation, where each road segment (= link) is represented as a first-in first-out queue with two restrictions [8, 9]: First, each agent has to remain for a certain time on the link, corresponding to the free speed travel time. Second, a link storage capacity is defined which limits the number of vehicles on the link; if it is filled up, no more agents can enter this link.

3. Evaluating Plans: All executed plans are evaluated by a utility function which in this paper encodes the perception of travel time and monetary costs for car and public transport. For the public transport mode, the utility function also accounts for waiting, access, and egress times. 
4. Learning: Some agents obtain new plans for the next iteration by modifying copies of existing plans. This modification is done by several strategy modules that correspond to the available choice dimensions. The choice between different plans is performed with respect to a multinomial logit model. As the number of plans is limited for every agent by memory constraints, adding a new plan to a person which already has the maximum number of plans permitted requires to discard one plan. From all plans of the choice set, including the newly obtained plan, the plan with the worst performance is discarded.

The repetition of the iteration cycle coupled with the agent database enables the agents to improve their plans over many iterations. This is why it is also called learning mechanism. The iteration cycle continues until the agents are assumed to have a plausible number of different plans in their choice set.

\subsection{Public Transport in MATSim}

Each public transport line in MATSim is defined by its mode, e.g. train/bus, the stops or stations vehicles will serve, the route each vehicle will ply, the vehicles associated with the line, and the departures of each of the line's vehicles. A public transport stop in MATSim is located at the end of a link. Agents using public transport can board and alight vehicles at stops only. Depending on the vehicle type, each boarding passenger and each alighting passenger delays the vehicle. The delay can be set for each type of vehicle. In addition, the vehicle's doors can operate in two different modes. First, the parallel mode allows simultaneous boarding and alighting at different doors. Thus, the total delay of the vehicle is defined by the maximum of the total boarding delay and the total alighting delay plus an additional delay for door operations. The second mode of operation is called serial; this mode is used whenever a door can be used by boarding as well as by alighting passengers with alighting passengers giving priority. The total delay of the vehicle is then the sum of total alighting delay and total boarding delay plus the additional delay of operating the doors. Another important attribute is the capacity of each vehicle. A vehicle fully loaded cannot pick up any more passengers, in which case passengers will have to wait for the next vehicle to arrive. Vehicles of one line can serve different tours. Consequently, the delay of one vehicle can be transferred to the following tour if the scheduled slack time at the terminus is insufficient to compensate this delay. Hence, agents not responsible for the delay in the first place are influenced in their experienced travel time and may be delayed as well. Further delays may occur by vehicle-vehicle interaction. In the case of mixed-traffic operation, private cars and buses compete for the same limited road capacity and thus can be caught in the same traffic jam. Each stop can be configured to either block traffic or to allow overtaking whenever a transit vehicle stops, i.e. a bus stop located at the curb will block traffic; if the bus can pull in a bus bay, other vehicles can pass. For an in-depth analysis of MATSim's public transport dynamics refer to [10] and [11].

\subsection{MATSim's Departure Time Adaptation}

In the present study, time is the only enabled choice dimension. During the iterative learning process, agents can adapt their departure times in order to extend, shorten or shift activities. Every iteration some agents are considered to generate and execute new plans, whereas the other agents choose among their existing plans. If an agent is considered for choice generation, a plan is randomly chosen from the agent's choice set. A replication of that plan is then modified by using a simple time 
allocation approach: For all activities of the plan, the end time (= departure time) is shifted by a random time period with a predefined maximum range. The actual time shift value will be a random draw between $0 \mathrm{~h}$ and the maximum value, i.e. allowing both for small and large shifts. The newly generated plan is then executed and evaluated. Time shifts that result in a higher utility are kept in the agent's choice set with a higher probability than time shifts that yield a lower utility. For instance, time shifts resulting in a departure time of the agent where there is no transit supply will result in a very low utility and are thus discarded immediately. Hence, it is crucial that the maximum time shift range will enable the agents to reach other potential departures within a single mutation step. Otherwise, the agents may stuck in a local optimum. In more complex scenarios, the time shift period of an agent might be limited by other constrains as opening and closing times of facilities. Thus, an agent might try to shift its departure by two hours but will discard this plan because the penalty for being too late at the working place does not compensate the shorter travel time. This rather simple departure time adaptation approach follows the KISS principle of avoiding all unnecessary complexity [12]. The following sections demonstrate that from this approach a more complex behavior of the traveling agents can emerge.

\section{Scenario}

\subsection{Supply}

For the simulation experiments we consider a single transit corridor with a total length of $1 \mathrm{~km}$. The network consists of two transit stops A and B that are located at both corridor's endpoints. Between 7 a.m. and 9 a.m. the corridor is served by a constant number of transit vehicles that run from A to B. The headway of this service is altered in each simulation experiment (see Section4). The transit vehicles are assumed to have an unlimited capacity; that is, occurrences of boarding denials can be excluded. The door operation mode is serial. Boarding and alighting times are set differently in each simulation experiment (see Section4). As the free speed is set to $36 \mathrm{~km} / \mathrm{h}$, the free travel time amounts to $100 \mathrm{sec}$. Alternative modes of transportation are not considered in this study. The agents are also not allowed to walk from transit stop A to B; that is, they have to use the public transport.

\subsection{Demand}

On the demand side, 2000 agents are considered. Each agent has two activity locations and one intermediate public transport trip. The first activity is located at transit stop A and the second one at transit stop B. During the simulation, agents adjust their departure times in order to shift, extend or shorten activity durations. The initial departure times are uniformly distributed from 7 a.m. to 9 a.m.

For evaluating the travel options a utility based approach is used. The total utility of an executed daily plan consists of a trip and an activity related utility:

$$
V_{p}=\sum_{i=1}^{n}\left(V_{\text {perf }, i}+V_{t r, i}\right)
$$

where $\mathrm{V}_{p}$ is the total utility of a plan; $\mathrm{n}$ is the total number of activity locations; $\mathrm{V}_{\text {perf }, i}$ is the (usually positive) utility for performing an activity $i$; and $\mathrm{V}_{t r, i}$ is the (usually negative) utility for traveling to activity $i$. The first and the last activity are handled as one activity, thus there are as many trips between activities as there are activities. The trip related utility is calculated as follows: 


$$
V_{t r, i}=\beta_{v, p t} \cdot t_{i, v, p t}+\beta_{w, p t} \cdot t_{i, w, p t}
$$

where $t i, v, p t$ is the in-vehicle time; $\beta v, p t$ is the marginal utility of the in-vehicle time $\left(-6\right.$ utils/h); ti,w,pt is the waiting time; and $\beta_{w, p t}$ is the marginal utility of waiting $(-6 \mathrm{utils} / \mathrm{h})$. For calculating the utility earned by performing an activity, a logarithmic form is used, thus, for longer activity durations the marginal utility is decreasing, but remains positive [13].

$$
V_{\text {perf }, i}=\beta_{\text {perf }} \cdot t_{*, i} \cdot \ln \left(\frac{t_{\text {perf }, i}}{t_{0, i}}\right)
$$

where tperf is the duration of an activity; $\mathrm{t} *$ is the "typical" duration of an activity $(12 \mathrm{~h})$; and $\beta_{\text {perf }}$ is the marginal utility of performing an activity at its typical duration ( +6 utils $/ \mathrm{h}) . t 0, i$ is a scaling parameter that has no effect as long as activities cannot be dropped from the plan.

The parameters used in this study are of synthetic nature and chosen in a way that the agents have enough time to spend at their activity locations. Thus, the differences in utility from extending or shortening the activity are very small. For more realistic scenarios the parameters for the marginal utility of performing an activity, traveling, waiting etc., can be replaced by estimated parameters that depict stated or revealed preferences [see, e.g., 6], or the parameters can be used to calibrate the model [see, e.g., 14]. Also for the typical duration of an activity, real world values can be used, e.g., 8 hours for the work activity. Note that the effective marginal utilities for in-vehicle and waiting times are obtained by adding the marginal opportunity cost of time to the base values. The opportunity cost of time is incurred from shortening the activity before or after the trip when a trip takes longer. The present investigation does not include a fare model and thus no marginal utility of money is given; otherwise, a (marginal) value of travel time savings would be given by dividing the effective marginal utilities of time by the marginal utility of money.

\section{Table 1. Overview of the Simulation Experiments of this Paper}

\begin{tabular}{rcc} 
& \multicolumn{3}{c}{ Number of iterations } \\
Headway & $100 \quad 200 \quad 1000 \quad 1100 \quad 1000010100$ \\
\hline $2 \mathrm{~min}$ & Each setting \\
$10 \mathrm{~min}$ & with and without \\
$60 \mathrm{~min}$ & boarding delay
\end{tabular}

\section{Simulation Experiments}

In this study the maximum number of plans per agent is set to 4. A plan is modified with a probability of $10 \%$. For the departure time adaptation, the maximum time shift period is set to $2 \mathrm{~h}$ (see Section 2.3). The simulation experiments are carried out for different iteration numbers, assumptions about the public transport service reliability and various headways. The complete overview of the 36 configurations of the simulation experiments can be seen in Table 1 .

Learning: Perfection vs. Imperfection We allow the learning mechanism to run for 100,1000 and 10000 iterations (see Section2.1). The adequate number of 
iterations usually depends on the agents' choice dimensions and the size of the scenario. Studies with less than 100 iterations are rather unusual, e.g., [15] and [16] use 400 to 2000 iterations for their case studies. Thus, a learning mechanism of 100 iterations is chosen as the starting point for further investigation. Common practice is to switch-off the creation of new plans after a certain number of iterations. In all three cases, 100 additional iterations are run without time allocation mutation. Agents then only choose among plans of their individual choice sets with respect to a multinomial logit model.

One of the research questions is to find out how many iterations are actually needed for the relaxation process to complete. As stated in the introduction, another question is under which conditions the agents over-adapt so that the model shows an unrealistic user behavior. For example, passengers may learn to arrive at the stop just in time to be able to board the vehicle the second before it departs. Furthermore, we will analyze the importance of running the simulation for a couple of iterations without plan modification, i.e. with fixed choice set.

Service reliability we consider two different cases: In the first case, we focus on departure time adaptation of passengers to a $100 \%$ reliable service. Therefore, the delays of transit vehicles due to passengers boarding/alighting are set to zero. The second setup assumes the public transport service to be less reliable. Boarding and alighting times are set to $1 \mathrm{sec}$ per person. Hence, actual travel times and headways can differ from the schedule. The agents randomly change their travel alternatives from iteration to iteration (random plan modification, plan selection according to a multinomial logit model). That is, the number of boarding agents per vehicle changes as well. Since, the delay of a transit vehicle depends on the number of boarding passengers, the delays will vary from iteration to iteration.

We will investigate how public transport reliability affects the passengers' travel behavior.

Headway variation: Considering typical urban situations, three different headways are simulated: 2 min which is common for important public transit lines during peak times, e.g., metro lines, $10 \mathrm{~min}$ which is a typical headway for less important public transit lines during peak times, and 60 min which is common for less important public transit lines, e.g., night buses. The headway refers to the scheduled time interval between transit vehicles that arrive at transit stop A.

We will analyze if and how the passengers' arrival patterns change with the headway. For the 2 min headway a random arrival at the transit stop seems plausible, whereas for the relatively long headway of $60 \mathrm{~min}$ a coordinated arrival seems more reasonable. The 10 min headway will presumably indicate the transition from random to coordinated arrival.

\section{Results}

\subsection{Learning: Perfection vs. Imperfection}

Up to iteration 100,1000 and $10000,10 \%$ of the agents are considered for experimental plan modification. These agents also have to execute the newly generated plan even though it may yield a much lower utility than the already existing plans of the agent's choice set. This might even result in an agent getting stuck, in case he/she is forced to depart after the last vehicle's departure. By running the simulation for an additional 100 iterations in which agents only choose among their existing plans, i.e., with a fixed choice set, this experimental behavior is 
excluded. The results show that with additional iterations agents do not miss the last departure.

Figure1illustrates the effect of the additional 100 iterations with fixed choice set. The histograms depict the agents' arrival times at the first stop A for the day time period from 7:30 to 8:30. The red line indicates the realized departure time of a transit vehicle. Both graphs show the results for the scenario with delay and $10 \mathrm{~min}$ headway. Figure 1a depicts the arrival times for iteration 1000. Figure 1 bshows the arrival pattern for the simulation experiment with 100 additional iterations with fixed choice set. Without fixing the choice set for additional 100 iterations, agents arrive within the first 5 minutes right after a transit vehicle's departure (see Figure 1a). These experimental plans are discarded in the following 100 iterations and thus are not present in the graph of iteration 1100 (Figure 1b). That is, in the simulation experiment with fixed choice set for the last 100 iterations (iteration 1100), the overall travel behavior seems to be much better adapted. However, a less adapted and more experimental travel behavior may be wanted by the modeler to reflect the imperfection of the real world travel behavior.

With more iterations, the agents have more time to adjust their travel behavior and the overall adaptation becomes more and more perfect. The resulting phenomenon of adaptation and possible over-adaptation is illustrated in Figure2. Again, the histograms refer to the passengers' arrivals at the first stop A. The three histograms show the results for the $60 \mathrm{~min}$ headway scenario without any delay induced by boarding and alighting agents. The graphs focus on the simulation outcome after additional 100 iterations without plan modification. In iteration 200, the majority of the agents pick an arrival time before the departure of the transit vehicle. Nevertheless, some agents

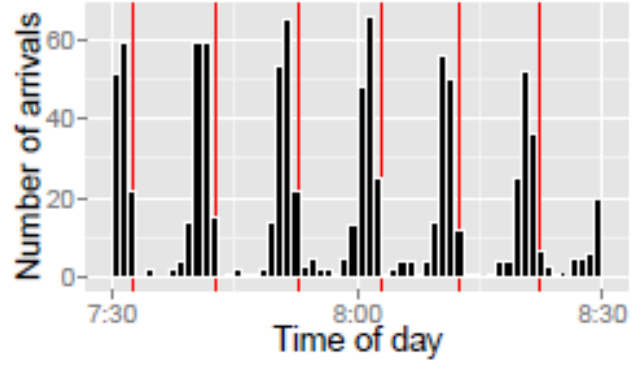

(a) Delay, 10 min, Iteration 1000

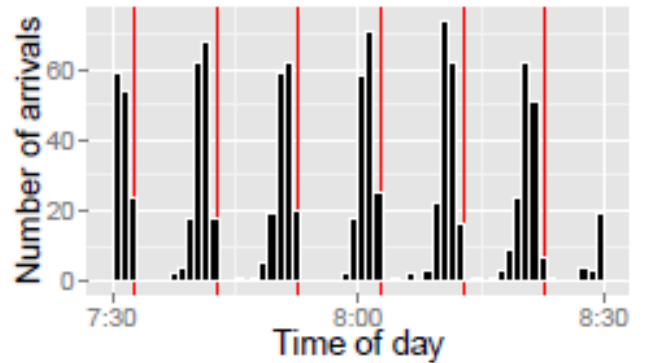

(b) Delay, 10 min, Iteration 1100

Figure 1. The Additional 100 Iterations without Plan Modification (with Fixed Choice Set) Remove Experimental Plans. The Results here were Gener- ated "with Delay" but Look Similar "without Delay"

arrive after 8 o'clock and are forced to wait for the next departure. In iteration 1100, the agents manage to cluster right before the departure of the vehicle. This becomes more extreme in iteration 10100 with all agents arriving within 2 minutes before the departure. However, in reality, this behavior is not possible due to a lack of perfect knowledge, i.e., departure times are unknown or congestion may prevent passengers from transferring as planned. Therefore, in terms of model calibration, it may make sense to stop departure time adaptation at an earlier stage to prevent agents from unrealistic behavior. 


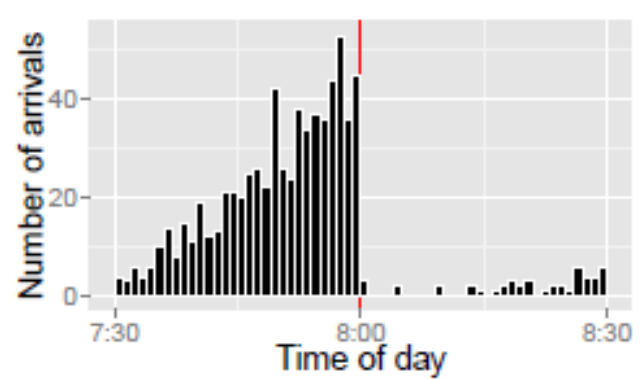

(a) No Delay, 60 min, Iteration 200

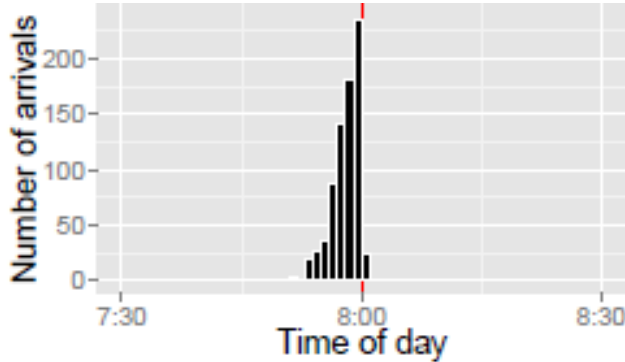

(b) No Delay, 60 min, Iteration 1100

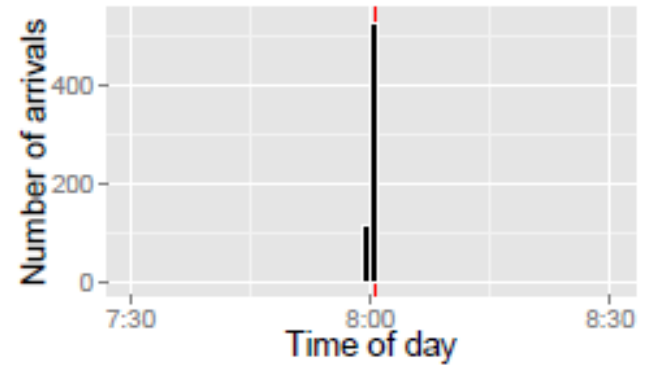

(c) No Delay, 60 min, Iteration 10100

Figure 2. More Iterations Directly Translate into Agents Over-optimizing

\subsection{Service Reliability}

Figure 3 depicts the passenger arrival pattern for a 60 min headway and 10100 iterations. In Figure $3 \mathrm{a}$ boarding and alighting times are set to zero and the 8:00 transit vehicle departs right on time. All agents arrive within two minutes before the departure. In contrast, in Figure $3 \mathrm{~b}$ boarding and alighting passengers are assumed to need time to board vehicles, and in the process to delay transit vehicles. Therefore, the vehicle needs more time to handle all boardings, and in consequence departs later. As indicated by the red line, the transit vehicle leaves at 8:16 instead of 8:00. When analyzing the simulation experiment with delayed transit vehicles (Figure 3b), two opposite effects are observed,

(1) Adaptation to delays As a first effect, passengers adapt their activity scheduling decisions according to the departure times of the transit vehicles. That is, many agents arrive at the stop well after the scheduled departure time. This effect becomes apparent

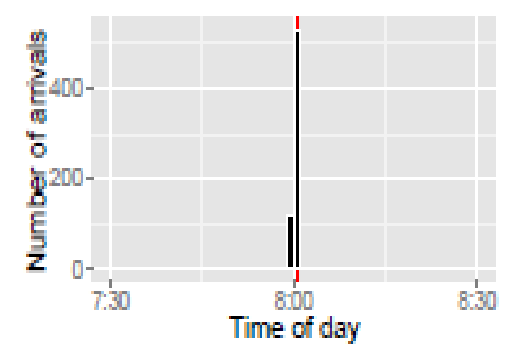

(a) No Delay, 60 min, Iteration 10100 (same as Figure 2c)

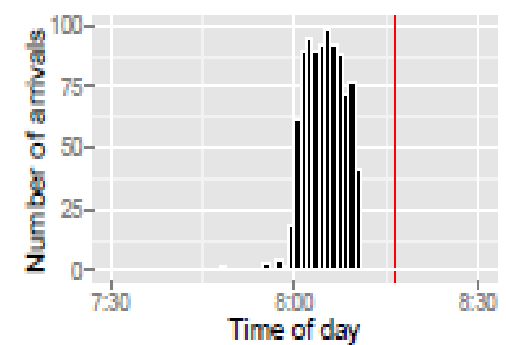

(b) Delay, 60 min, Iteration 10100

\section{Figure 3. Modeling the Boarding Delay Results in a more Realistic Arrival Pattern at the Stop}

in Figure $3 \mathrm{~b}$ showing agents arriving after the scheduled departure time of 8:00. The agents' choice sets function as a memory that allows for adaptation according to an experienced schedule. Agents start to incorporate the experienced delay of the 
transit service into their daily plans. Hence, passengers arrive late on purpose well knowing that the vehicle is still at the stop handling other passengers. Only few agents are arriving for the scheduled departure time, i.e., before 8:00. According to the utility functions and parameter settings described in Section 3.2, agents that arrive late spend more time at the previous activity location and therefore earn a higher positive utility. Additionally, they face shorter waiting times and therefore a higher trip related utility.

(2) Adaptation to unreliability As a second effect, agents also adapt their departures according to the experienced reliability of the schedule. This effect is shown in Figure 3 bwhere no agents arrive at the transit stop right before the realized departure time (gap between black bars and red line). The strategy of incorporating the exact vehicle delays becomes futile if transit vehicles display different delays from one iteration to the next. In case the queue of boarding passengers is interrupted, the transit vehicle departs and agents arriving later need to wait for the next departure. Therefore, the strategy depends on a considerably large number of agents to reliably delay the departure. Fewer agents before one's own arrival increase the risk of missing a particular transit vehicle. As mentioned earlier, arriving at the latest moment yields the shortest travel time and thus the highest utility. At the same time, it increases the period of time some other agents need to delay the vehicle and thus increases the risk of being stranded. As a consequence, the agents increase the reliability of their plan by adding a buffer, i.e., agents arrive well before the delayed departure.

The agents' risk aversion depends on the headway, i.e. the time until the next transit vehicle arrives in case a departure is missed. For the $10 \mathrm{~min}$ headway (see Figure 1), the number of arriving passengers at the transit stop is observed to decrease right before the transit vehicle departs. Most of the agents prefer to arrive earlier to ensure not to miss the departure. For the 2 min headway, the risk aversion is irrelevant and buffers are not present (not shown).

\subsection{Public Transport Headways}

The literature review indicates a more coordinated arrival pattern for larger headways. MATSim's simple plan modification strategy is able to reflect this as shown in Figure4:

All three graphs show the period of time between two departures for a headway of $60 \mathrm{~min}, 10 \mathrm{~min}$, and $2 \mathrm{~min}$. In the $60 \mathrm{~min}$ headway scenario, the arrivals accumulate towards the departure. In the $10 \mathrm{~min}$ headway scenario, this effect becomes less clear. Whereas in the 2 min headway scenario, the arrivals are more or less equally distributed. 


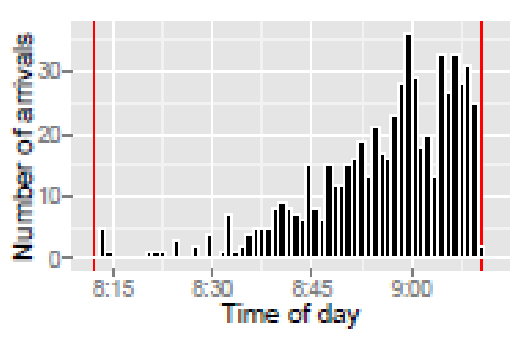

(a) Delay, 60 min, Iteration 200

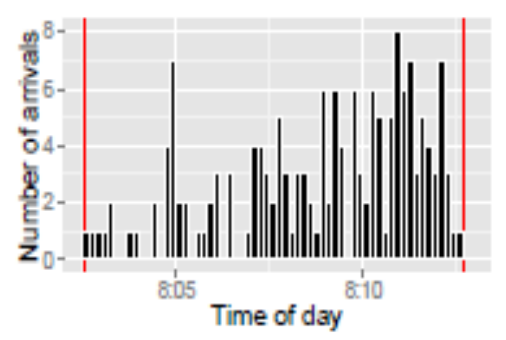

(b) Delay, 10 min, Iteration 200

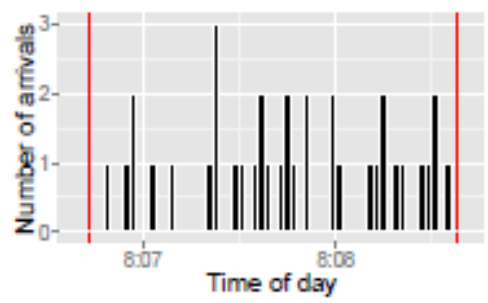

(c) Delay, 2 min, Iteration 200

\section{Figure 4. Larger Headways Result in more Coordinated Arrival Patterns}

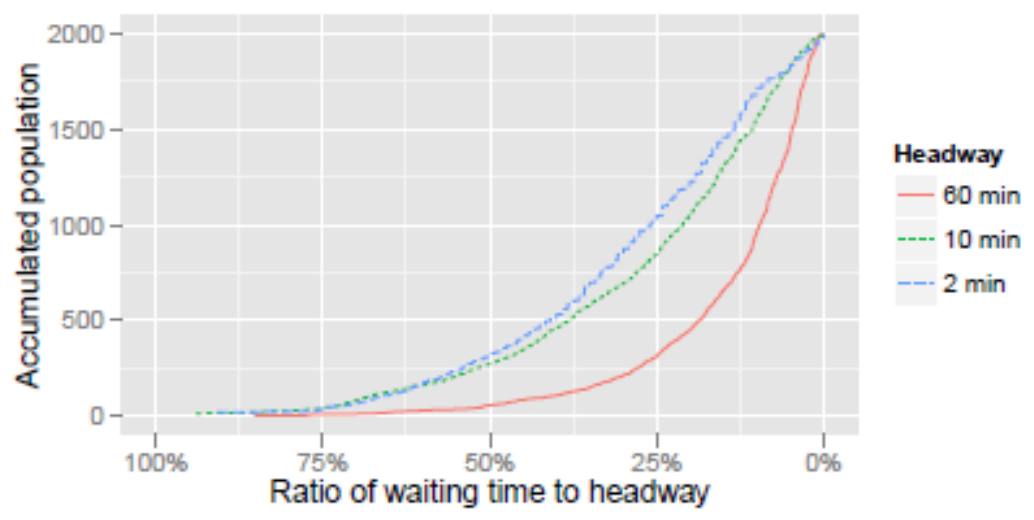

Figure 5. Waiting Time Distribution of the Complete Population for each Headway (Delay, Iteration 200)

This effect becomes even clearer when analyzing the complete population over all de- partures: Figure 5 shows the distribution of the waiting time considering all departures. Agents waiting as long as the headway have a ratio of waiting time to headway of $100 \%$. Agents arriving just in time have a ratio of $0 \%$. For the $60 \mathrm{~min}$ scenario, the arrivals are coordinated with the departure of the vehicle, i.e., over 84 $\%$ of the agents arrive $15 \mathrm{~min}$ before the departure or even later. In the other scenarios, the arrivals are less coordinated, e.g., in the 2 min scenario, less than 50 $\%$ of the agents arrive $30 \mathrm{sec}$ or less before the departure.

The simulation experiments indicate that the simple random time allocation module together with a realistic scoring function is indeed able to show the trend of real world effects: Random passenger arrivals for short headways; timed passenger arrivals for larger headway.

The explanation for the observed arrival patterns starts with the way travel alternatives are chosen from the agents' choice sets, i.e., the multinomial logit model. For all headways, plans are generated following the same method, which yields the same probability for each departure time to be added to the choice set. For smaller head- ways, the relative utility differences of plans with randomly shifted departure times is small since missing a departure means only little additional waiting time for 
the next vehicle; in consequence, the probability of the multinomial logit model to choose such a plan is still fairly high. Therefore, from iteration to iteration travel behavior is more variable and the experienced schedule is less reliable. In consequence, passengers arrive more randomly at stops. Contrarily, for larger headways, the plan modification results in longer waiting times for the next departure. Thus, the relative utility differences are larger. In this case, the probability of the multinomial logit model to choose such a plan is much lower, and thus the plan with the highest utility is executed more constantly.

The diversity of travel alternatives is also related to the way in which the agents' choice set is modified over the iterations. As described in Section2.1, in case an agent's choice set contains already the maximum number of permitted plans per agent and this agent generates a new plan (with modified departure times), from all plans in the choice set, including the newly generated plan, the plan with the worst performance is removed. This may lead to fairly similar plans and explains the observed over-optimization, i.e., for longer simulation runs (see Figure2c).

\section{Discussion}

An approach to increase the diversity in travel behavior is the path size logit model which gives a penalty to plans that are very similar to others [e.g., 17]. In this paper we propose a rather simple approach that removes a plan once it is found to be similar to another plan of the choice set. If no plan is considered similar to another plan the standard MATSim behavior is preserved as described in Section 2.1. A plan is considered similar to another plan if all similarity checkers consider the plan similar. In this paper, only the activity end times are checked for. That is, two plans with the same activity end times are considered similar. Depending on the use case, the proposed approach can be extended and further similarity checkers can be introduced. Additional similarity checkers may for example include the mode of transportation, the route or the activity locations. From two plans considered as similar the older one is preserved and the newer one will be deleted.

As first tests indicate, this effectively prevents agents from over-adapting, i.e., arriving at a stop only seconds before the vehicle departs. For instance, Figure 6 shows the results of the proposed approach for the $60 \mathrm{~min}$ headway. There, plans are considered similar if the activity end times lie within an interval of 5 minutes. The 5 minutes are motivated by the observed tendency that passengers do not plan their trips to the exact minute. Again, agents are allowed to adapt and show a coordinated arrival pattern similar to the one shown in Figure 2. However, the comparison of iteration 1100 and 10100 indicates that the adaptation stops at a certain level and no over-adaptation occurs.

Another issue is that this study assumes the capacity of transit vehicles to be unlimited. This was done for the purpose of removing all interfering effects; the software itself is capable of including the effect of finite vehicle sizes. The same passenger behavior as in the present study will be observed in cases where boarding denials due to capacity constraints are not the limiting factor, e.g., for modes with large vehicle sizes like train services or for areas where passengers can avoid an over-subscribed transit service by taking a different transit line or changing the mode of transport.

In case boarding denials caused by capacity constraints are the limiting factor, the passenger behavior will be different. In the extreme case where the sum of all bus depar- 


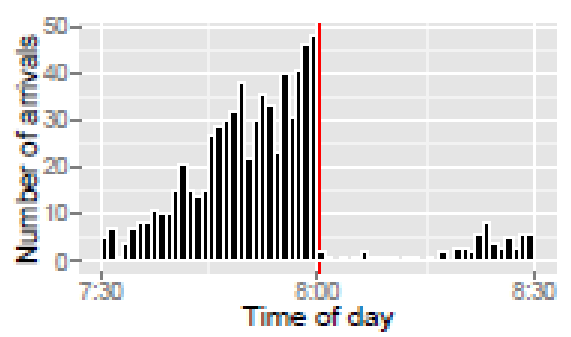

(a) No Delay, 60 min, Iteration 200

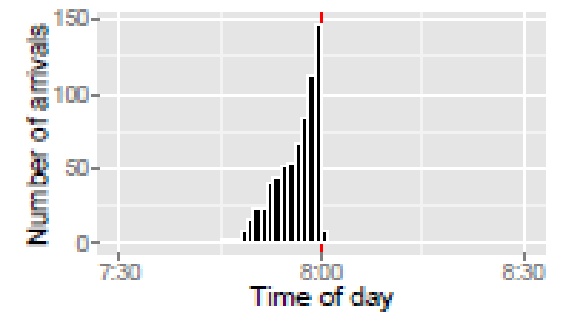

(b) No Delay, 60 min, Iteration 110

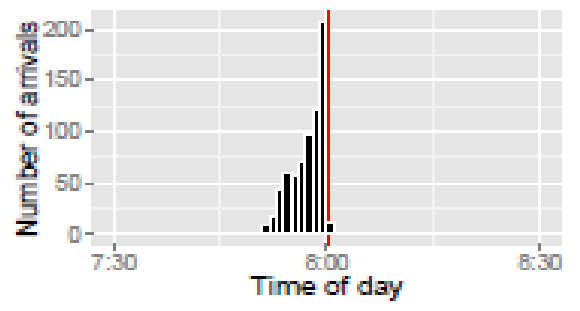

(c) No Delay, 60 min, Iteration 10100

\section{Figure 6. Removing Similar Plans from the Choice Set Effectively Prevents Agents from Over-optimizing}

tures has less capacity then there is demand, the score of an agent will be dominated by the agent's ability to reach its destination at all. In consequence, assuming a firstcome first-serve policy at the station, the agents are forced to arrive as early as possible at the transit stop. Depending on its position in the waiting queue at the station, the agent would either be able to board one of the buses, or not. The actual vehicle would matter much less than the capability to board at all, and being earlier at the stop than the competing agents would be the only thing that matters.

\section{Conclusion}

This study investigates arrival patterns of passengers at transit stops within the frame- work of an agent-based simulation. Different simulation experiments are carried out for a simple transit corridor. Passenger arrival patterns are analyzed focusing on three aspects: the agents' degree of learning, the service reliability, and the headway.

It is demonstrated that complex behavior can emerge from the simple adaptation approach of MATSim: Agents try to obtain the latest possible arrival time at the transit stop in order to minimize the waiting time. Agents incorporate the experienced delay of the vehicle and arrive late on purpose well knowing that the vehicle is still handling other passengers. Adding delay effects imposed by boarding/alighting passengers also affects the reliability of the public transport service: Besides being behind schedule, the actual departure time of a vehicle varies from iteration to iteration depending on the number of boarding passengers. This less reliable service induces a second effect of how agents adapt their activity scheduling decisions: Agents increase the reliability of their plans by adding a buffer time between their arrival at the stop and the actual departure of the vehicle.

More iterations are observed to translate directly into better adapted users. Depending on the scenario configuration, agents need a different number of adaptation iterations to show a realistic arrival pattern: For a reliable transit service, fewer iterations might better reflect the imperfection of real world travelers. For a less reliable service, more iterations are required to model the users' adaptation to the experienced vehicle departures, eventually reflecting real world travel behavior in a better way. Simulating an additional 100 iterations without plan modification (with fixed choice set) removes ex- perimental/imperfect travel alternatives. A 
simple approach is proposed that modifies the model such that realistic arrival patterns are obtained in the steady state of the iterations, irrespective of the number of previous iterations.

The results are consistent with the literature on arrival patterns for different headways. Smaller headways yield a more equally distributed arrival pattern. Larger headways result in more coordinated arrival patterns. That is, the realistic passenger arrival patterns at transit stops emerge endogenously from the simulation.

\section{References}

[1] J. K. Jolliffee and T. P. Hutchinson. "A Behavioural Explanation of the Association Between Bus and Passenger Arrivals at a Bus Stop", Transportation Science, vol. 9, no. 3, (1975), pp. 248282.

[2] L. A. Bowman and M. A. Turnquist, "Service frequency, schedule reliability and passenger wait times at transit stops", Transportation Research Part A: General, vol. 15, no. 6, (1981), pp. 465471.

[3] M.-D. Salek and R. B. Machemehl, "Characterizing bus transit passenger wait times", Tech. rep. Center for Transportation Research, University of Texas at Austin, (1999).

[4] M. Luethi, U. Weidmann and A. Nash, "Passenger arrival rates at public stations", 86th Transportation Research Board Annual Meeting, (2007).

[5] PTV AG. VISUM 12.5 Grundlagen. 2012. url: http://vision-traffic.ptvgroup.com/enus/products/ptv-visum/.

[6] I. Kaddoura, B. Kickhöfer, A. Neumann and A. Tirachini. "Agent-based opti- misation of public transport supply and pricing: Impacts of activity scheduling decisions and simulation randomness", Transportation, ISSN: 0049-4488, doi: 10.1007/s11116-014-9533-6, vol. 42, no. 6, (2015), pp. 1039-1061.

[7] B. Raney and K. Nagel, "An improved framework for large-scale multi-agent simulations of travel behaviour", Towards better performing European Transportation Systems. Ed. by P. Rietveld, B. Jourquin, and K. Westin. London: Routledge, (2006), pp. 305-347.

[8] C. Gawron. "An Iterative Algorithm to Determine the Dynamic User Equilibrium in a Traffic Simulation Model", International Journal of Modern Physics C, vol. 9, no. 3, (1998), pp. 393-407.

[9] N. Cetin, A. Burri and K. Nagel, "A Large-Scale Agent-Based Traffic Microsimulation Based On Queue Model", Proceedings of the Swiss Transport Research Conference (STRC). See www.strc.ch. Earlier version, with inferior performance values: Transportation Research Board Annual Meeting 2003 paper number 03- 4272. Monte Verita, Switzerland, url: http://www.strc.ch, (2003).

[10] A. Neumann and K. Nagel, "Avoiding bus bunching phenomena from spreading: A dynamic approach using a multi-agent simulation framework", VSP Working Paper 10-08. See www.vsp.tu-berlin.de/publications. TU Berlin, Transport Systems Planning and Transport Telematics, 2010. url: https://svn.vsp.tu-berlin.de/repos/public-svn/publications/vspwp/2010/1008 .

[11] M. Rieser. "Adding transit to an agent-based transportation simulation concepts and implementation", PhD thesis, Technische Universität Berlin, 2010. urn: urn: nbn:de:kobv:83opus-27852.

[12] R. M. Axelrod, "The complexity of cooperation: Agent-based models of competition and collaboration", Princeton University Press, (1997).

[13] D. Charypar and K. Nagel, "Generating complete all-day activity plans with genetic algorithms", Transportation, vol. 32, no. 4, (2005), pp. 369-397.

[14] A. Neumann, M. Balmer and M. Rieser, "Converting a Static Trip-Based Model Into a Dynamic Activity-Based Model to Analyze Public Transport Demand in Berlin", Travel Behaviour Research: Current Foundations, Future Prospects. Ed. by M.J. Roorda and E.J. Miller. International Association for Travel Behaviour Research (IATBR), Chap. 7, isbn: 9781304715173, (2014) February, pp. 151-176.

[15] A. Neumann. "A paratransit-inspired evolutionary process for public transit net- work design", PhD thesis. Technische Universität Berlin, 2014. urn: urn:nbn:de: kobv:83-opus4-53866.

[16] B. Kickhöfer, "Economic Policy Appraisal and Heterogeneous Users", PhD thesis, Technische Universität Berlin, 2014. urn: urn:nbn:de:kobv:83-opus4-53489.

[17] M. Ben-Akiva and M. Bierlaire, "Discrete Choice Methods and their Applications to Short Term Travel Decisions”, ed. by R. Hall. Kluwer, (1999), pp. 5-34. 


\section{Authors}

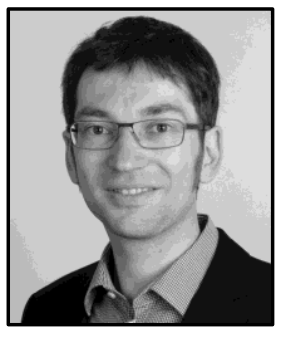

Andreas Neumann received a Diploma in Transport Planning and Transport Operation and holds a PhD in Transport Modeling. He is currently working as a project manager at Senozon and as a guest researcher at Technische Universität Berlin at the Trans- port Systems Planning and Transport Telematics research group of Kai Nagel. His main research interests comprise of evolutionary algorithms, game theory, heuristics, traffic engineering, and pub- lic transport operations including the transit network design and optimal transport supply modeling.

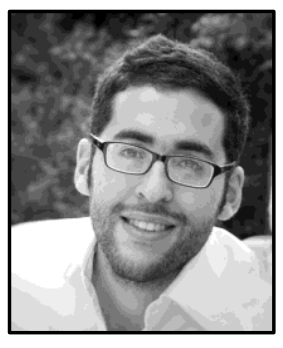

Ihab Kaddoura is a research associate and $\mathrm{PhD}$ student at the Transport Systems Planning and Transport Telematics research group at Technische Universität Berlin. He received a Diploma in Industrial Engineering and Management, majoring in traffic planning and traffic control. His main research interests com- prise of dynamic and agent-based transport models, user oriented optimization of private and public transport systems, transport related externalities, and pricing.

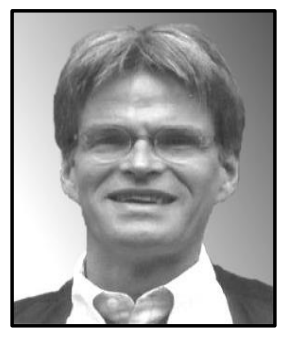

Kai Nagel is full professor for Transport Systems Planning and Transport Telem5ics at Technische Universität Berlin. He was trained in physics and climate research, and holds a $\mathrm{PhD}$ in informatics from University of Cologne, Germany. He worked at Los Alamos National Laboratory, USA, from 1995 to 1999, at ETH Zürich from 1999 to 2004, and holds his current post since 2004. His research interests include large-scale transportation simula- tions, modeling and simulation of socio-economic systems, and largescale computing. 